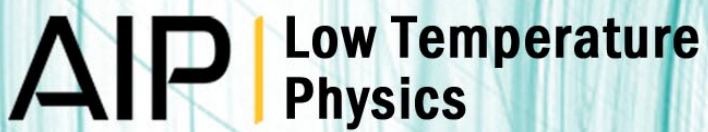

\section{Perpendicular magnetic anisotropy in Co-Pt granular multilayers}

J. Bartolomé, A. I. Figueroa, L. M. García, F. Bartolomé, L. Ruiz et al.

Citation: Low Temp. Phys. 38, 835 (2012); doi: 10.1063/1.4752096

View online: http://dx.doi.org/10.1063/1.4752096

View Table of Contents: http://ltp.aip.org/resource/1/LTPHEG/v38/i9

Published by the American Institute of Physics.

\section{Related Articles}

Effective magnetic anisotropy of annealed FePt nanoparticles

Appl. Phys. Lett. 101, 172402 (2012)

Light-induced magnetization reversal of high-anisotropy TbCo alloy films

Appl. Phys. Lett. 101, 162408 (2012)

Enhanced microwave absorption in columnar structured magnetic materials

J. Appl. Phys. 112, 083908 (2012)

Metastable state in a shape-anisotropic single-domain nanomagnet subjected to spin-transfer-torque Appl. Phys. Lett. 101, 162405 (2012)

Influence of chemical pressure in Sn-substituted Ni2MnGa Heusler alloy: Experimental and theoretical studies J. Appl. Phys. 112, 073921 (2012)

\section{Additional information on Low Temp. Phys.}

Journal Homepage: http://ltp.aip.org/

Journal Information: http://ltp.aip.org/about/about_the_journal

Top downloads: http://ltp.aip.org/features/most_downloaded

Information for Authors: http://ltp.aip.org/authors

\section{ADVERTISEMENT}

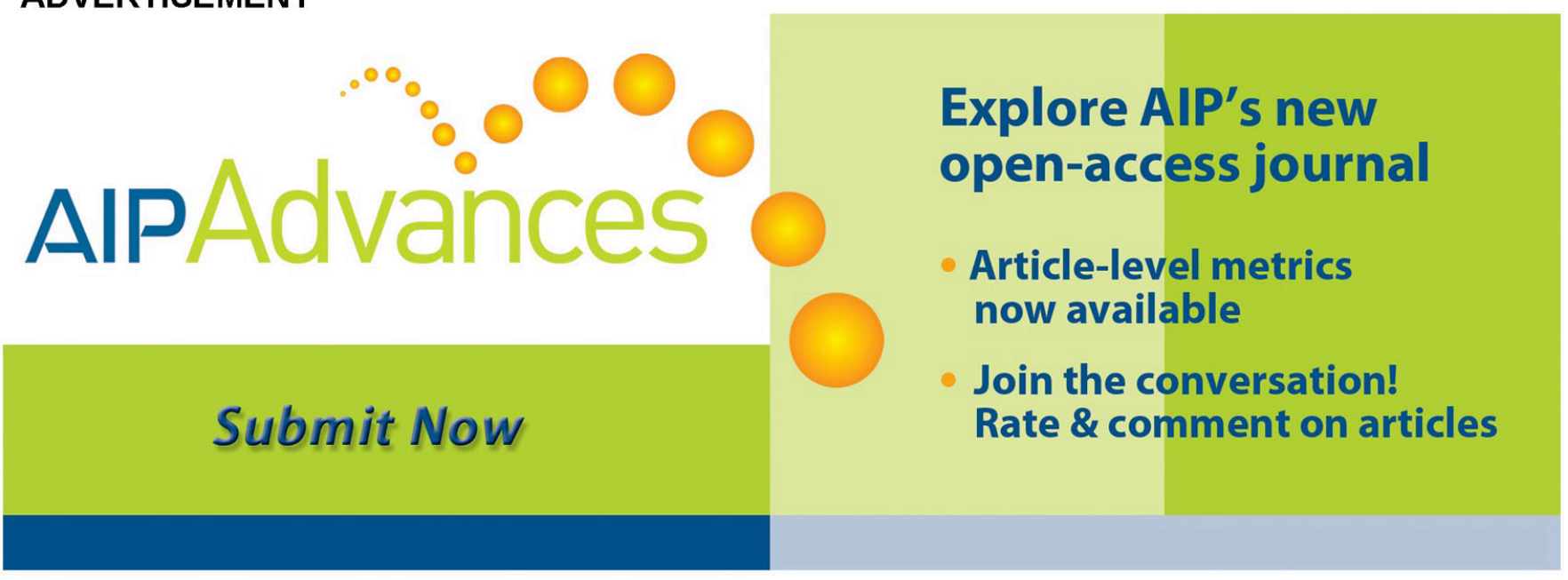




\title{
Perpendicular magnetic anisotropy in Co-Pt granular multilayers
}

\author{
J. Bartolomé, a) A. I. Figueroa, L. M. García, and F. Bartolomé \\ Instituto de Ciencia de Materiales de Aragón, Departamento de Física de la Materia Condensada CSIC/U de \\ Zaragoza, Zaragoza, Spain
}

L. Ruiz and J. M. González-Calbet

Departamento de Química Inorgánica, Universidad Complutense de Madrid, E-28040 Madrid, Spain

\section{F. Petroff and C. Deranlot}

Unité Mixte de Physique, CNRS/Thales, Route Départementale 128, 91767 Palaiseau Cedex, France and Université Paris-Sud, 91405 Orsay (Paris), France

\section{F. Wilhelm, A. Rogalev, and N. Brookes}

European Synchrotron Radiation Facility, BP220, F-38043 Grenoble, France (Submitted April 20, 2012)

Fiz. Nizk. Temp. 38, 1053-1057 (September 2012)

\begin{abstract}
Magnetization hysteresis curves have been measured on Co granular multilayers, $\left(\mathrm{Al}_{2} \mathrm{O}_{3} / \mathrm{Co} / \mathrm{Pt}\right)_{N}$ $(N=1$ and 25), with the applied magnetic field parallel and perpendicular to the substrate plane. In all samples perpendicular magnetic anisotropy was observed. For Co particles with average diameter $3 \mathrm{~nm}$, the coercive field at low temperature is $\mu_{0} H_{C}=0.5 \mathrm{~T}$. $H_{C}$ decreases for increasing temperature and disappears at $\approx 200 \mathrm{~K}$. A soft magnetic component is also present in all samples up to the freezing temperature $T_{f}=365 \mathrm{~K}$. Co and Pt XMCD measurements at the $L_{2,3}$ edges were performed, yielding to the orbital $m_{L}$ and spin $m_{S}$ contributions to the total magnetic moment of the system. These results, in addition to XANES ones, indicate the presence of $\mathrm{Co}_{x} \mathrm{Pt}_{1-x}$ alloy. Particles conformed of CoPt alloy, embedded in Pt and coupled magnetically by dipolar or RKKY interaction, may explain the phenomenology observed in these systems. C 2012 American Institute of Physics. [http://dx.doi.org/10.1063/1.4752096]
\end{abstract}

\section{Introduction}

Granular Co thin film multilayers constitute a family of nanoparticle systems which have been very useful for understanding size effects in magnetism. The reason is that Co clusters of nearly spherical shape can be produced by sputtering of Co on amorphous alumina, previously deposited on a Si substrate. Capping with a noble metal enables to modify the matrix that surrounds the particle, thus also modifying its magnetic properties. When capping with $\mathrm{Cu}, \mathrm{Ag}$ or $\mathrm{Au}$ the particles behave as superparamagnetic with anisotropy constants that depend on the metal used. ${ }^{1-3}$ Instead, capping with Pt has a completely different effect. In this case Co particles are strongly coupled via the polarized Pt. ${ }^{4}$ Besides, in a recent work ${ }^{5}$ a small increase in anisotropy was detected on Co particles capped with $\mathrm{Pt}$, which was related to the formation of $\mathrm{CoPt}_{3}$ clusters. In this paper we show that the Co-Pt granular multilayers present strong perpendicular magnetic anisotropy (PMA) to the substrate plane.

PMA has been observed in $\mathrm{Co} / \mathrm{Pt}$ thin film multilayers, when the Co thickness is below a threshold Co thickness of $11 \mathrm{ML}$, and has been assigned to a strong increase in the surface magnetocrystalline anisotropy. ${ }^{6}$ On the other hand, $\mathrm{Co}_{1-x} \mathrm{Pt}_{x}$ alloy films have also shown PMA. ${ }^{7}$ In the case of (111) $\mathrm{CoPt}_{3}$ thin films, the PMA was established by means of X-ray magnetic circular dichroism (XMCD) measurements at both the Co and the Pt $L_{2,3}$ edges. They could correlate to the existence of anisotropic structural effects induced during the codeposition process. ${ }^{8}$ So, the origin of PMA may be associated to interface or alloying effects, or both. In the present work we show that in the case of Co nanoparticles capped with $\mathrm{Pt}$, alloying plays the dominant role.

\section{Experimental}

\subsection{Sample preparation and morphology}

The samples measured in this work were prepared by sequential sputtering on a $\mathrm{Si}$ substrate at room temperature. The multilayers are formed by a number $N$ of $\left(\mathrm{Al}_{2} \mathrm{O}_{3} / \mathrm{Co} / \mathrm{Pt}\right)$ trilayers, each formed by a buffer layer of alumina, another layer of $\mathrm{Co}$, and a third of Pt. The Co coalesces into clusters such that the mean particle diameter is proportional to the nominal thickness, $t_{\mathrm{Co}}$, of the deposited Co. In this work we present results on samples with $t_{\mathrm{Co}}=0.7 \mathrm{~nm}$, which produces Co particles of $3 \mathrm{~nm}$ average diameter when deposited on amorphous alumina. Two series of multilayers, with $N=1$ and 25 have been measured. ${ }^{1}$ The capping Pt film has $t_{\mathrm{Pt}}=1.5 \mathrm{~nm}$ depth and the separation between layers was $t_{\mathrm{Al}_{2} \mathrm{O}_{3}}=3 \mathrm{~nm}$ (see Fig. 1 for $N=25$ ). The capping metal tends to alloy with the Co particle and fills the interparticle spaces with the excess $\mathrm{Pt}$, as can be observed in the Fig. 1 and inset.

\subsection{Magnetic measurements}

Magnetization measurements were performed with a SQUID magnetometer equipped with the high resolution option. For $t_{\mathrm{Co}}=0.7 \mathrm{~nm}$ the $M(T)$, measured with a bias field $\mu_{0} H_{d c}=0.02 \mathrm{~T}$ in the direction parallel to the substrate plane, shows a maximum at the freezing temperature $T_{f}=365 \mathrm{~K}$ (see also Fig. 2). The $M(H)$ were measured with the field applied parallel and perpendicular to the substrate plane at 


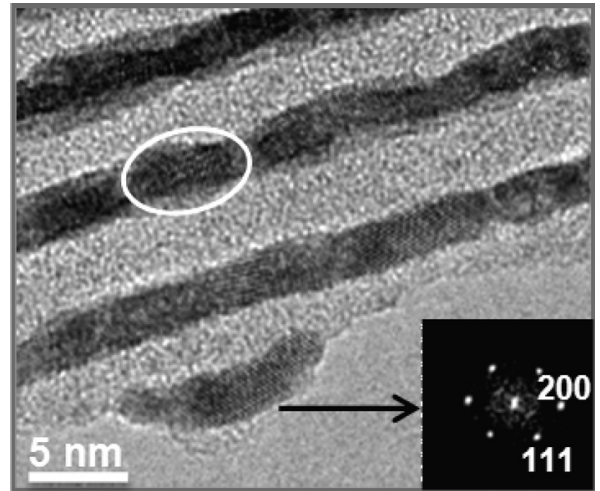

FIG. 1. TEM cross-section of a granular multilayer with $N=25, t_{\mathrm{Co}}$ $=0.7 \mathrm{~nm}, t_{\mathrm{Pt}}=1.5 \mathrm{~nm}$, and $t_{\mathrm{Al}_{2} \mathrm{O}_{3}}=3 \mathrm{~nm}$ (intermediate stripes of light grey). Inset: Fourier transform showing Pt crystalline morphology in the selected area. The circle indicates a Co-Pt particulate morphology.

several temperatures. In Fig. 3 we show the data for $t_{\text {Co }}$ $=0.7 \mathrm{~nm}$ and $N=1$ measured at $T=5 \mathrm{~K}$. Above $T_{f}$ the curves have the characteristic superparamagnetic behavior, which can be fitted to a Langevin curve of the nominal Co particle average diameter. ${ }^{4}$ At low temperatures the hysteresis curves measured in the perpendicular direction have a characteristic step near $H=0$, and a second step when the switching to the reverse orientation takes place (Fig. 3). The former step indicates that there is a magnetically soft component present in the sample, while the latter shows the presence of a ferromagnetic hard component with a rather high coercive field $\mu_{0} H_{C}=0.5 \mathrm{~T}$ at $T=5 \mathrm{~K} . H_{C}$ decreases with temperature till it disappears at about $200 \mathrm{~K}$ (Fig. 4). On the other hand, in the direction parallel to the substrate plane there is no coercive field. The ferromagnetic behavior implies that the Co-Pt particles are strongly coupled. Besides, the spontaneous direction of the anisotropy is perpendicular to the plane. Therefore, it is proven unambiguously that just one layer of granular Co capped with Pt has a strong PMA below $200 \mathrm{~K}$.

From 200 till $300 \mathrm{~K}$ the ferromagnetic soft component subsists till its disappearance at $T_{f}$. It is interesting to note that upon annealing the sample up to $800 \mathrm{~K}$ the hard magnetic component transforms into the soft component, so that the coercivity is strongly reduced (down to $0.055 \mathrm{~T}$ ) when remeasured at low temperatures.

The multilayer (sample with $N=25$ ) shows the same overall behavior as the sample with $N=1$ regarding the

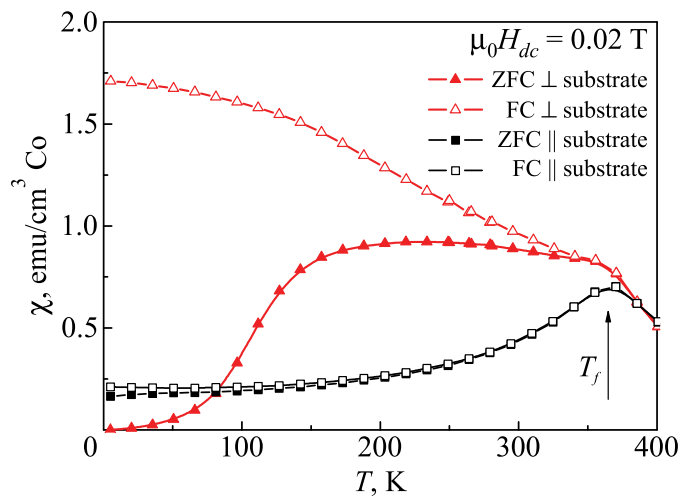

FIG. 2. Magnetization as a function of temperature measured for a trilayer of $\left(\mathrm{Al}_{2} \mathrm{O}_{3} / \mathrm{Co} / \mathrm{Pt}\right) N=25, t_{\mathrm{Co}}=0.7 \mathrm{~nm}$ and $t_{\mathrm{Pt}}=1.5 \mathrm{~nm}$, with a bias field of $\mu_{0} H_{d c}=0.02 \mathrm{~T}$. $(\mathbf{\Delta}, \triangle) H$ perpendicular to the substrate, $(\square, \square) H$ parallel to the substrate.

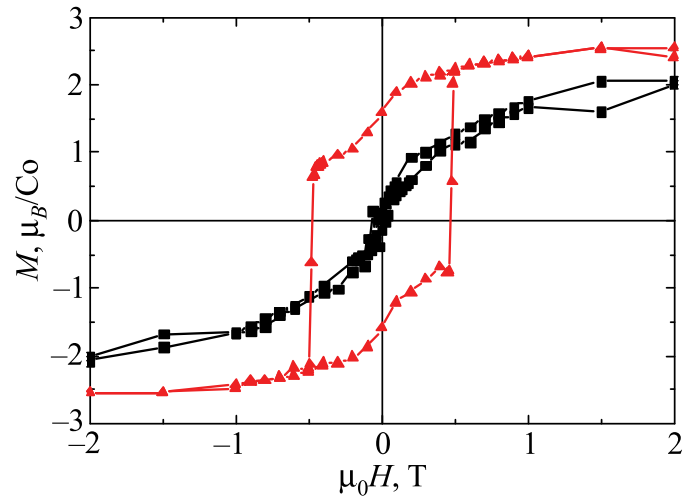

FIG. 3. Hysteresis loops at $T=5 \mathrm{~K}$ for a trilayer of $\left(\mathrm{Al}_{2} \mathrm{O}_{3} / \mathrm{Co} / \mathrm{Pt}\right) N=1$, $t_{\mathrm{Co}}=0.7 \mathrm{~nm}$ and $t_{\mathrm{Pt}}=1.5 \mathrm{~nm}$. (山) $H$ perpendicular to the substrate, (ם) $H$ parallel to the substrate.

PMA (see Fig. 5) and coercivity decay with temperature (see Fig. 4). However the soft magnetic component is not as evident as it is in the sample with $N=1$.

\section{3. $X M C D$ measurements}

The XMCD results provide evidence of hybridization between the $\mathrm{Co}$ at the particle surface and the capping metal in the cases of $\mathrm{Cu}$ and Au-capping. ${ }^{1}$ Similarly, the presence of hybridization between $\mathrm{Co}$ and $\mathrm{Pt}$ was reported earlier. ${ }^{4}$ In this work the Co and $\mathrm{Pt} L_{2,3}$ edges XMCD results are presented. The Co XMCD measurements were performed at the ESRF ID08 instrument in total electron yield detection, and the Pt ones at the ID12 with fluorescence detection.

From an XMCD experiment at the $L_{2,3}$ edges of Co or Pt one obtains, through the sum rules, ${ }^{9}$ the spin $m_{S}$ and orbital $m_{L}$ moments averaged over the whole sample, provided one knows by calculation or estimation the number of holes, $n_{h}$, existing involved in the $2 p \rightarrow n d$ excitations ( $n=3$ and 5, for Co and Pt, respectively). For Co we have taken the $n_{h}$ values proposed in the case of $\mathrm{CoPt}_{3}$ thin films, ${ }^{8}$ and for $\mathrm{Pt}$ we have derived $n_{h}$ from the white line difference with a Pt foil at the $L_{2,3}$ edges XANES (see Table 1 ).

XMCD experiments at the Co $L_{2,3}$ edges as a function of incident angle $\left(0^{\circ}\right.$ and $\left.60^{\circ}\right)$ were performed at $T=5 \mathrm{~K}$ and $\mu_{0} H=1 \mathrm{~T}$, looking for anisotropy of the orbital moment. However, the results did not show clear angle dependence, which was unexpected. In Fig. 6 the XMCD spectrum for normal incidence is shown. These spectra have been normalized

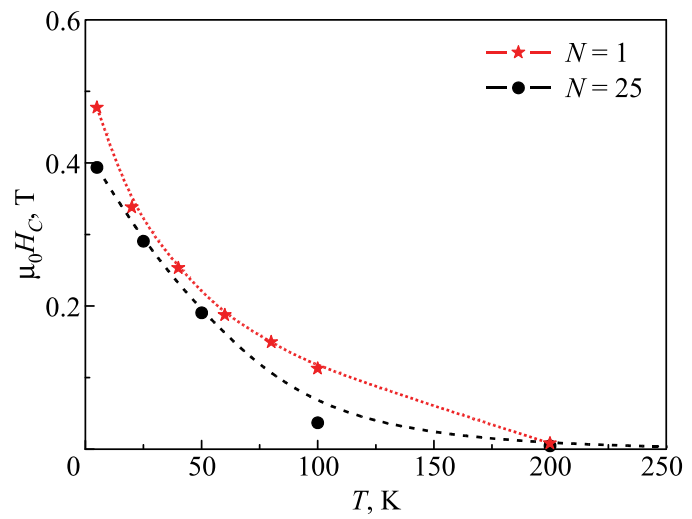

FIG. 4. Coercive field of the hard magnetic component in sample $\left(\mathrm{Al}_{2} \mathrm{O}_{3} /\right.$ $\mathrm{Co} / \mathrm{Pt}) N=1$ and $25, t_{\mathrm{Co}}=0.7 \mathrm{~nm}$ and $t_{\mathrm{Pt}}=1.5 \mathrm{~nm}$. 


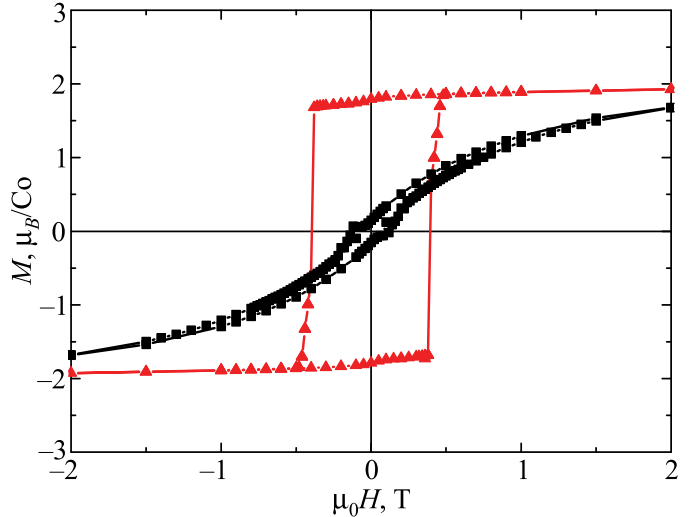

FIG. 5. Hysteresis loops at $T=5 \mathrm{~K}$ for a multilayer of $\left(\mathrm{Al}_{2} \mathrm{O}_{3} / \mathrm{Co} / \mathrm{Pt}\right) N=$ $25, t_{\mathrm{Co}}=0.7 \mathrm{~nm}$ and $t_{\mathrm{Pt}}=1.5 \mathrm{~nm}$. (ム) $H$ perpendicular to the substrate, $H$ parallel to the substrate.

to the X-ray absorption spectra (XAS) in high-energy limit, after background subtraction. The value of the Co orbital moment obtained $m_{L}=0.21 \mu_{B}$ (see Table 1) resembles that found for $\mathrm{CoPt}_{3}$ films deposited on a substrates at $800 \mathrm{~K}$, which also showed absence of anisotropy, ${ }^{8}$ and that of annealed $\mathrm{Co}_{0.5} \mathrm{Pt}_{0.5}$ NPs with diameters of about $2.6 \mathrm{~nm}$ deposited on amorphous carbon matrices. ${ }^{10}$ The spin moment $m_{S}=1.98 \mu_{B}$ has been obtained considering that the dipole term is small with respect to the total $m_{S}$. The value found is larger than that found in $\mathrm{CoPt}_{3}$ films $\left(m_{S}=1.44 \mu_{B}\right)$ but close to that of the annealed $\mathrm{Co}_{0.5} \mathrm{Pt}_{0.5} \mathrm{NPs}\left(m_{S}=1.91 \mu_{B}\right)$.

In a recent work, XMCD measurements at the Co $L_{2,3}$ edges on $\mathrm{Co}$ and Co-Pt particles have been reported. ${ }^{11,12}$ They find systematically larger ratios of $m_{L} / m_{S}$ for comparable particle sizes. The orbital to spin ratio $m_{L} / m_{S}$ of Co atoms in our Co-Pt NPs is 0.11 , which is close to the value found for particles of $2.0 \mathrm{~nm}$ with composition close to $\mathrm{Co}_{3} \mathrm{Pt}^{12}$

The XMCD spectra measured at the $\mathrm{Pt} L_{2,3}$ edges are shown in Fig. 7 and the data extracted from them are included in Table 1. The non-zero values measured reflect the polarization of the $\mathrm{Pt}$ by the magnetic Co. The values found are in reasonable agreement with those found for $\mathrm{CoPt}_{3}$, again. ${ }^{8}$ The induced moment on $\mathrm{Pt}$ is one order of magnitude larger than the moments induced on the $\mathrm{Cu}, \mathrm{Ag}$ or $\mathrm{Au}$ capping cases. $^{2}$ Obviously, the reason for this difference is the proximity of $\mathrm{Pt}$, with the $5 d^{9}$ band, to fulfill the Slater criterion for ferromagnetism, instead of the nearly filled noble metal $n d^{10}$ bands ( $n=3,4$ and 5 for $\mathrm{Cu}, \mathrm{Ag}$ or $\mathrm{Au})$.

\section{Conclusion}

The presence of PMA has been detected in all $\left(\mathrm{Al}_{2} \mathrm{O}_{3} /\right.$ $\mathrm{Co} / \mathrm{Pt})_{N}$ multilayers, independently of being $N=1$ or 25 . For the $t_{\mathrm{Co}}=0.7 \mathrm{~nm}$ samples the switching field of one layer is

TABLE 1. The Co and Pt orbital and spin moments for sample with $N=25$.

\begin{tabular}{lcc}
\hline \hline Parameter & Co $L_{2,3}$ edge & Pt $L_{2,3}$ edge \\
\hline$n_{h}$ & $2.25^{\mathrm{a}}$ & $1.80(2)$ \\
$m_{L} / m_{S}$ & $0.11(1)$ & $0.19(1)$ \\
$m_{L}, \mu_{B}$ & $0.21(1)$ & $0.027(1)$ \\
$m_{S}, \mu_{B}$ & $1.98(2)$ & $0.14(1)$ \\
$m, \mu_{B}$ & $2.20(2)$ & $0.16(1)$ \\
\hline \hline
\end{tabular}

${ }^{\mathrm{a}} n_{h}$ : number of holes taken from Ref. 8 .

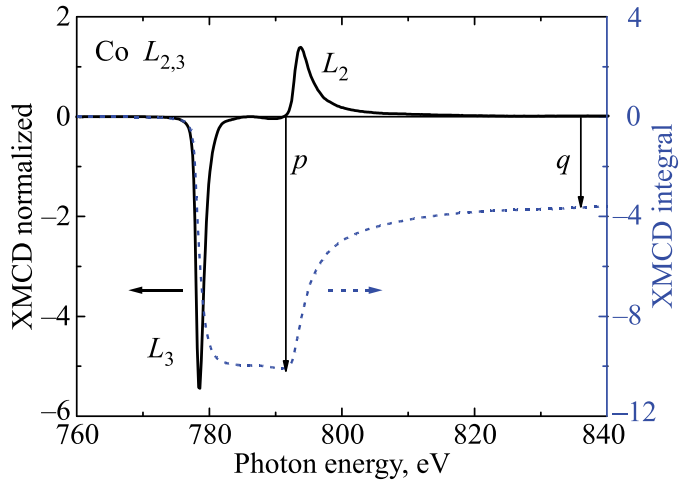

FIG. 6. XMCD spectra at the Co $L_{2,3}$ edge of the sample at $T=5 \mathrm{~K}$ and $H$ $=1 \mathrm{~T}$, at normal incidence for a multilayer $\left(\mathrm{Al}_{2} \mathrm{O}_{3} / \mathrm{Co} / \mathrm{Pt}\right) N=25, t_{\mathrm{Co}}=$ $0.7 \mathrm{~nm}$ and $t_{\mathrm{Pt}}=1,5 \mathrm{~nm}$. Dashed line is the integrated area of the spectra.

$\mu_{0} H_{S} \approx 0.5 \mathrm{~T}$ and the maximum coercive field measured at low temperatures is $\mu_{0} H_{C}=0.52 \mathrm{~T}$.

The $\mathrm{Pt}$ capping by sputtering on the Co particles preformed on $\mathrm{Al}_{2} \mathrm{O}_{3}$ gives rise to a quite different behavior than when the same particles are capped with noble metals. The magnetic behavior is that of ferromagnetic thin films below $T_{C}=365 \mathrm{~K}$ (when $t_{\mathrm{Co}}=0.7 \mathrm{~nm}$ ), instead of blocked superparamagnetic behavior. There is evidence that there are two different contributions, a soft and a hard magnetic component, the latter with a Curie temperature of circa $200 \mathrm{~K}$. From Co $K$-edge XAS measurements it was proven that there has been Co-Pt alloying in the capping process, ${ }^{3}$ since the XANES spectra differ markedly from the metallic Co one, and are very similar to that of $\mathrm{Co}_{x} \mathrm{Pt}_{1-x}{ }^{13}$

Thus, $\mathrm{Co}_{x} \mathrm{Pt}_{1-x}$ alloys are present in all the samples studied. These alloys are known to have a variety of magnetic properties depending on their composition and crystallinity. ${ }^{14,15}$ For example, the crystallographically ordered alloy $\mathrm{CoPt}_{3}$ has a Curie temperature of $T_{C}=290 \mathrm{~K}$. A departure from the exact composition or lack of perfect crystallinity would reduce it to $T_{C} \approx 200 \mathrm{~K}$, which is the temperature of disappearance of coercivity in our samples. Therefore, one may identify the PMA appearing on this material as due to the formation of $\mathrm{Co}_{x} \mathrm{Pt}_{1-x}$ alloy with short range ordering in the $L 1_{0}$ structure. When annealed, the induced disorder destroys these crystallites and the coercivity disappears. The XMCD data also point towards the same explanation, by comparison to measurements on different Co-Pt systems. Thus, a $\mathrm{Co}_{x} \mathrm{Pt}_{1-x}$ alloy is probably surrounding the Co rich core, in view of the particulate morphology of the Co-Pt layers observed by TEM. The Co rich core of the sample

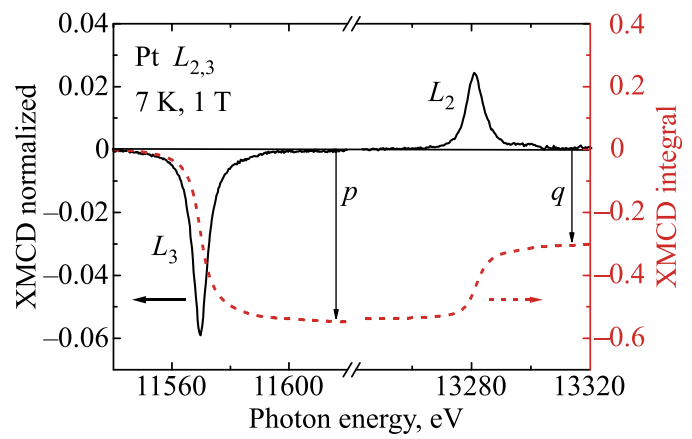

FIG. 7. XMCD spectra at the Pt $L_{2,3}$ edges for a trilayer of $\left(\mathrm{Al}_{2} \mathrm{O}_{3} / \mathrm{Co} / \mathrm{Pt}\right) N$ $=25$, and $t_{\mathrm{Co}}=0.7 \mathrm{~nm}, t_{\mathrm{Pt}}=1.5 \mathrm{~nm}$ measured at $7 \mathrm{~K}$ and $1 \mathrm{~T}$. 
would be responsible for the soft magnetic component in the magnetic hysteresis.

The peak observed in the $M(T)$ curve at $T_{f}=365 \mathrm{~K}$ (Fig. 2) could be related to the magnetic coupling of the particles which form in each layer a bidimensional array of ferromagnetic particles. As it was described earlier, $T_{f}$ depends on the average particle size, and above $T_{f}$ the magnetic behavior is superparamagnetic. Therefore, the overall picture describing the collective behavior of these multilayers is that of $\mathrm{Co}_{x} \mathrm{Pt}_{1-x}$ alloyed particles, embedded in the non-reacted Pt, strongly coupled via the polarized $\mathrm{Pt}$, via dipolar or RKKY interaction. Then, the short range order within the grains would create the anisotropy in the $\mathrm{Co}_{x} \mathrm{Pt}_{1-x}$ alloy which would give rise to the PMA.

Moreover, the sample preparation may play an important role, as demonstrated from comparison with results of Co-Pt NPs systems prepared by different chemical and physical methods. ${ }^{10-12}$

It can also be concluded from this work that the search for Co-Pt hybridization surface effects that was the leitmotif of this work, might be present but are overcome by the $\mathrm{Co}_{x} \mathrm{Pt}_{1-x}$ alloying in the magnetic properties.

This work was supported by the MECOM under Grant MAT11/23791 and Project IMANA from the DGA, in Spain. A.I.F. acknowledges a CSIC JAE2008-Predoc grant. The experiment HE3136 at the ESRF is acknowledged.

${ }^{\text {a)} E m a i l: ~ b a r t o @ u n i z a r . e s ~}$

${ }^{1}$ F. Luis, F. Bartolomé, F. Petroff, J. Bartolomé, L. M. García, C. Deranlot, H. Jaffres, M. J. Martínez, P. Bencok, F. Wilhelm, A. Rogalev, and N. B. Brookes, Europhys. Lett. 76, 1 (2006).
${ }^{2}$ J. Bartolomé, L. M. García, F. Bartolomé, F. Luis, R. LópezRuiz, F. Petroff, C. Deranlot, F. Wilhelm, A. Rogalev, P. Bencok, N. B. Brookes, L. Ruiz, and M. González-Calbet, Phys. Rev. B 77, 184420 (2008).

${ }^{3}$ J. Bartolomé, F. Luis, L. M. García, F. Bartolomé, F. Petroff, C. Deranlot, F. Wilhelm, A. Rogalev, P. Bencok, and N. B. Brookes, Mater. Sci. Forum 570, 1 (2008).

${ }^{4}$ J. Bartolomé, L. M. García, F. Bartolomé, F. Luis, F. Petroff, C. Deranlot, F. Wilhelm, and A. Rogalev, J. Magn. Magn. Mater. 316, e9 (2007). Note that the value of $m_{L} / m_{S}=0.10$ given in this reference is now changed to $m_{L} / m_{S}=0.22$.

${ }^{5}$ J. Arbiol, F. Peiro, A. Cornet, C. Clavero, A. Cebollada, G. Armelles, and Y. Huttel, Appl. Phys. Lett. 86, 032510 (2005).

${ }^{6}$ W. B. Zeper, F. J. A. M. Greidanus, P. F. García, and G. R. Fincher, J. Appl. Phys. 65, 4971 (1989).

${ }^{7}$ D. Weller, H. Brändle, G. Gorma, C.-J. Lin, and H. Notarys, Appl. Phys. Lett. 61, 2725 (1992).

${ }^{8}$ W. Grange, M. Maret, J. P. Kappler, J. Vogel, A. Fontaine, F. Petroff, and G. Krill, Phys. Rev. B 58, 6298 (1998).

${ }^{9}$ P. Carra, P. T. Thole, M. Altarelli, and X. Wang, Phys. Rev. Lett. 70, 694 (1993); B. T. Thole, P. Carra, F. Sette, and G. van der Laan, Phys. Rev. Lett. 68, 1943 (1992).

${ }^{10}$ F. Tournus, N. Blanc, A. Tamion, P. Ohresser, A. Perez, and V. Dupuis, J. Electron. Spectrosc. Relat. Phenom. 166-167, 84 (2008).

${ }^{11}$ P. Imperia, P. Andreazza, D. Schnitz, J. Penuelas, and C. AndreazzaVignole, J. Magn. Magn. Mater. 310, 2417 (2007).

${ }^{12}$ P. Imperia, L. Glaser, M. Martins, P. Andreazza, J. Penuelas, V. Alessandrovic, H. Weller, C. Andreazza-Vignolle, and W. Wurth, Phys. Status Solidi A 205, 1047 (2008).

${ }^{13}$ Y. S. Lee, J. Y. Rhee, C. N. Wang, and Y. P. Lee, Phys. Rev. B 68, 235111 (2003).

${ }^{14}$ F. Bolzoni, F. Leccabue, R. Panizzieri, and L. Pareti, IEEE Trans. Magn. 20, 1625 (1984).

${ }^{15}$ D. C. Martin, J. Phys. F: Met. Phys. 5, 1031 (1975).

This article was published in English in the original Russian journal. Reproduced here with stylistic changes by AIP. 